\title{
PROJETO CANANÉIA: ATIVIDADE DE EXTENSÃO UNIVERSITÁRIA QUE INTEGRA GRADUAÇÃO, SERVIÇO E COMUNIDADE
}

\author{
PROJECT CANANÉIA: ACADEMIC EXTENSION ACTIVITY THAT \\ INTEGRATES GRADUATION, SERVICE AND COMMUNITY
}

*Antônio Carlos Frias, **Simone Rennó Junqueira, ***Celso Zilbovicius e ****Maria Ercilia de Araujo

\section{RESUMO}

A extensão universitária é o processo educativo, cultural e científico que viabiliza a relação transformadora entre a universidade e a sociedade. Este trabalho é um relato da experiência do Projeto Cananéia, idealizado em I992 e coordenado desde 2007 pelo Departamento de Odontologia Social da Faculdade de Odontologia da USP. Propõe-se um conjunto de atividades de atenção primária para que os alunos conheçam a política de saúde municipal e as condições de vida da população, promovendo ações de educação, levantamento epidemiológico e atendimento odontológico básico. A aproximação com a realidade sensibiliza o aluno sobre a importância dessas ações, fornecendo subsídios para uma melhor formação acadêmica, com estímulo para a reflexão crítica em relação ao seu próprio processo formativo.

Palavras-chave: Relações comunidade-instituição. Atenção primária à saúde. Saúde bucal.

\begin{abstract}
Academic extension is an educational, cultural and scientific process that articulates teaching and research in an indivisible way and enables the transforming relation between university and society. This is an experience report of The Cananéia Project (southern coast of the State of São Paulo), idealized in 1992 by the undergraduate students of the São Paulo State University Dental School (FO-USP). The project proposes a set of primary oral health care activities. It is expected that students get acquaintance with the health policy of Cananéia; check the living conditions of the target population; conduct epidemiological survey in oral health; provide primary oral health care and promote actions of oral health education. Permitting the students to get acquainted with the real condition of health of the population can evoke on them a more conscious approach of the importance of these practices, providing support to a better academic background. This also serves as a stimulus for a critical reflection about their learning process.
\end{abstract}

Key words: Community-institutional relations. Primary health care. Oral health.

\footnotetext{
* Professor doutor do Departamento de Odontologia Social da Faculdade de Odontologia da Universidade de São Paulo - Av. Prof. Lineu Prestes, 2227, Cidade Universitária-São Paulo-SP - 05508-900-e-mail: acfrias@usp.br; srj@usp.br. ** Professora doutora do Departamento de Odontologia Social da Faculdade de Odontologia da Universidade de São Paulo. *** Professor doutor do Curso de Especialização em Saúde Coletiva da Fundação para o Desenvolvimento Gientífico e Tecnológico da Odontologia, da Faculdade de Odontologia da Universidade de São Paulo - e-mail: mocel@uol.com.br. **** Professora associada do Departamento de Odontologia Social da Faculdade de Odontologia da Universidade de São Paulo - e-mail: mercilia@usp.br
} 


\section{INTRODUÇÃO}

A extensão universitária é o processo educativo, cultural e científico que articula o ensino e a pesquisa de forma indissociável e viabiliza a relação transformadora entre a universidade e a sociedade [I4]. Tratase de uma via de mão dupla, com trânsito assegurado à comunidade acadêmica, que encontrará, na sociedade, a oportunidade de elaboração da práxis de um conhecimento acadêmico.

No retorno à universidade, docentes e discentes trarão um aprendizado que, submetido à reflexão teórica, será acrescido àquele conhecimento. Esse fluxo, que estabelece a troca de saberes sistematizados, acadêmico e popular, terá como consequências a produção do conhecimento resultante do confronto com a realidade brasileira e regional, a democratização do conhecimento acadêmico e a participação efetiva da comunidade na atuação da universidade. Além de instrumentalizadora deste processo dialético de teoria/ prática, a extensão é um trabalho interdisciplinar que favorece a visão integrada do social.

O Projeto Cananéia é uma das atividades de extensão da Faculdade de Odontologia da Universidade de São Paulo (FO-USP), concebida pelos próprios alunos do curso de graduação. A idealização deste Projeto data de meados de I992, por meio de contatos com acadêmicos de outras unidades da USP na área da saúde, que já desenvolviam atividades na região do Vale do Ribeira.

A escolha de Cananéia pela FO-USP levou em conta o fato de que, na época, não havia nenhum cirurgião-dentista contratado ou concursado na rede pública de saúde. Além disso, por ser uma cidade de grande pobreza, seria ambiente bastante instrutivo para a realização de um projeto de extensão como sua definição.

\section{CARACTERIZAÇÃO DO MUNICÍPIO}

O município de Cananéia, localizado na Região Administrativa de Registro, conhecida como Vale do Ribeira, é um dos primeiros povoados existentes no Brasil. A chegada dos primeiros colonizadores europeus na região data do início do século XVI, com figuras importantes como Américo Vespúcio, Gonçalo Coelho, entre outros. Oficialmente, considera-se que a fundação da cidade ocorreu em I2 de agosto de I53I, com a chegada da expedição portuguesa comandada por Martim Afonso de Souza.
Hoje, o lugar é terra de índios, caiçaras, quilombolas e mestiços, todos herdeiros de um território pobre marcado pela natureza exuberante, antigo marco do Tratado de Tordesilhas, e por preservar uma arquitetura típica do Brasil Colônia, com suas fachadas "sem eira nem beira", portas de madeira estreitas e altas e grandes janelas.

É no município de Cananéia que se localiza o Parque Estadual da Ilha do Gardoso (PEIC), reserva ambiental criada pelo Decreto n. 40.3I9, de 3 de julho de 1962, que abrange uma área de I5.IOO hectares, onde são encontrados todos os tipos de vegetação da Mata Atlântica costeira, proporcionando uma variedade extraordinária de ambientes e alta diversidade biológica.

A região de proteção é constituída por seis comunidades caiçaras, que, com forte influência cultural indígena, desenvolveram um apurado conhecimento da natureza. Formadas em sua maioria por pescadores, atualmente, têm o turismo como fonte substancial de renda [I2].

$\mathrm{Na}$ faixa litorânea, a pesca exerce papel fundamental na ocupação e no desenvolvimento econômico das comunidades locais. Os principais produtos comercializados pelos pescadores são o camarão e a ostra, além de crustáceos e pescados [9].

No entanto, a principal atividade econômica e fonte de renda da população do Vale do Ribeira é a agricultura, sendo as culturas mais presentes nas lavouras a banana e o chá preto, que ocupam áreas mais extensas e têm maior relevância do ponto de vista comercial.

Com área de $1.242 \mathrm{~km}^{2}$, o município abriga, atualmente, I4.299 habitantes, com uma densidade demográfica de $10,9 \mathrm{hab} . / \mathrm{km}^{2}$. A população com menos de 15 anos representa $28,4 \%$ do total e aquela com mais de 60 anos responde por 9,6\%. A taxa de natalidade é de I4, 9 nascimentos por mil habitantes e a taxa de mortalidade na infância corresponde a I 4,4 óbitos por mil nascidos vivos. Já o analfabetismo atinge IO, $9 \%$ da população com mais de I5 anos [I5].

Quanto aos indicadores de saúde bucal, em $2006,8,9 \%$ da população teve acesso à primeira consulta odontológica, I7,2\% receberam a escovação supervisionada e 59, 9\% estavam cobertos pelo Programa Saúde da Família - PSF [6].

Segundo o Cadastro Nacional de Estabelecimentos de Saúde, Cananéia possui um PSF na microárea Marujá, um na microárea de Ariri; uma unidade de PSF em 
Itapitangui e uma unidade mista [5]. Ainda havia duas cirurgiãs-dentistas contratadas pela prefeitura municipal.

\section{HISTÓRICO DO PROJETO CANANÉIA}

Em meados de 1992, em função de contatos entre acadêmicos da odontologia com outros de unidades da USP da área da saúde que já desenvolviam projetos na região do Vale do Ribeira, surgiu o interesse de participar de alguma atividade que viesse a contribuir para a melhora da saúde bucal da população.

O trabalho de campo iniciou-se em 3I de janeiro de I993, com o reconhecimento do município de Cananéia e o estabelecimento de contato com a Secretaria de Saúde e de Educação. Contava-se com os apoios da Pró-Reitoria de Cultura e Extensão Universitária da USP e da FO-USP, os quais se mantêm.

Em abril de I993, I2 alunos da FO-USP fizeram um levantamento por amostragem em 854 estudantes de 6 a I2 anos das escolas municipais da área urbana, o que revelou um índice CPOD (média de dentes cariados, perdidos e obturados) de 6,75 , a partir do qual o modo operacional do projeto foi direcionado para que pudesse, dentro das condições existentes, reverter a alta incidência e prevalência de cárie.

Em outubro do mesmo ano, I6 alunos iniciaram as atividades de promoção de saúde, atendendo apenas as crianças autorizadas pelos responsáveis. Devido à escassez de subsídios financeiros, em I998, estabeleceuse também uma parceria com a empresa Colgate-Palmolive, que destinou recursos para a continuidade do projeto e possibilitou sua expansão para a zona rural e ribeirinha, por vezes atendendo até a população adulta.

Entre 1993 e 2005 , foram realizadas atividades semestrais com uma equipe formada por aproximadamente 20 estudantes, dois docentes e dois cirurgiõesdentistas. Foram visitadas escolas e diversas comunidades do município, como as populações caiçaras do Cambriú, Enseada da Baleia e Maruja, localizadas na Ilha do Cardoso; a Vila do Ariri, situada na Baía de Paranaguá; a comunidade quilombola do Mandira, localizada na Estrada do Itapitangui; e a comunidade Santa Maria, na zona rural.

\section{PROPOSTAS DE ATUAÇÃO}

Em 2007, o Departamento de Odontologia Social da FO-USP assumiu a coordenação do projeto, com propostas de adequar a ação como uma atividade de extensão universitária. A extensão viabiliza a aproximação do conhecimento produzido na universidade com a realidade social, podendo estabelecer uma relação entre condição de vida com produção científica e formação profissional, a fim de compreender e transformar as relações sociais a que homens e mulheres estão submetidos.

Até 2005 , as atividades desenvolvidas apresentavam caráter predominantemente voltado para a saúde do escolar, com pouca ênfase na compreensão da dinâmica social local e no diálogo e pactuação de projetos comuns com a comunidade.

Em junho de 2007, a fim de reorganizar a atividade, reuniram-se professores de Saúde Coletiva, cirurgiões-dentistas (ex-participantes em edições do Projeto Cananéia), estudantes da FO-USP e a Secretaria Municipal de Saúde (SMS) de Cananéia para uma reflexão sobre a história da atividade, a condição socioeconômica e a organização dos serviços de saúde municipais. A partir deste momento, delinearam-se outros rumos para o Projeto Cananéia, estabelecendo-se novos objetivos e metodologias de trabalho, com foco num processo de ensino-aprendizagem crítico.

Dentro deste processo, é importante que os alunos da FO-USP vivenciem a saúde em seu sentido ampliado no contexto municipal.

Em 2008, foram realizadas duas imersões nas comunidades do Distrito de Ariri, na Ilha do Cardoso e na Vila de Santa Maria: a primeira entre 16 e 22 de março; e a segunda de 29 de junho a 05 de julho. No ano seguinte, as atividades se desenvolveram no período de 5 a II de abril e de 22 a 29 de agosto.

Pretende-se, ao longo dos próximos anos, atender, de acordo com o levantamento de necessidades da população, as famílias residentes no Distrito do Ariri e Vila de Santa Maria, localizados na área continental de Cananéia, e as comunidades ribeirinhas da Vila de Maruja, Enseada da Baleia e Pontal do Leste, na Ilha do Cardoso (Tabela I, ver anexo). A ampliação da cobertura de atendimento para famílias de outras comunidades do município dependerá da resolutividade das ações previstas pelo Projeto Cananéia e da resolutividade do próprio serviço de saúde do município.

Como atividade de extensão de cunho pedagógico e a partir de um processo de diálogo entre equipe do projeto, SMS e comunidade, pretende-se especificamente:

- conhecer a política de saúde do município de Cananéia; 
- verificar as condições de vida da população-alvo;

- realizar levantamento das necessidades odontológicas;

- proporcionar atendimento odontológico básico para os adultos e crianças da comunidade;

- estimular o envolvimento do grupo de estudantes com a comunidade atendida;

- promover ações de educação em saúde junto à comunidade;

- estimular um mecanismo de referência dos usuários para o serviço de saúde municipal;

- realizar pesquisas científicas em saúde de abordagem qualitativa e quantitativa;

- estimular a reflexão crítica dos estudantes em relação ao processo de formação do cirurgião-dentista.

\section{OPERACIONALIZAÇÃO DO TRABALHO DE CAMPO}

\section{RECURSOS HUMANOS}

Para possibilitar as ações, é formado um grupo de trabalho com dois estudantes do segundo ano de graduação, seis estudantes do terceiro/quarto ano de graduação, seis estudantes do quinto/sexto ano de graduação e equipe técnica (composta por um professor de saúde coletiva e coordenador do projeto, dois professores de odontologia da FO-USP e dois a três alunos do curso de pós-graduação ou cirurgiões-dentistas). Após o processo seletivo dos estudantes, os escolhidos passam por treinamento específico elaborado pela equipe técnica.

\section{PROCESSO PEDAGÓGICO}

Para que o grupo de estudantes compreenda a saúde em seu sentido ampliado, utiliza-se a pedagogia da problematização $[\mathrm{I}, 8]$. São realizados grupos de discussão, observação exploratória do território, entrevistas com moradores locais e leituras de texto, alternados com os períodos de atendimento odontológico, com o objetivo de estimular a reflexão crítica dos estudantes em relação ao processo de formação do cirurgião-dentista. Cada aluno anota as informações coletadas num diário de campo.

Os estudantes desenvolvem ações assistenciais correspondentes às competências e ao ano de graduação em curso. As atividades educativas são realizadas por todos os membros da equipe. Todas as ações são supervisionadas pela equipe técnica do projeto e por cirurgiões-dentistas do quadro de profissionais do município.

\section{ESTRUTURA FÍSICA}

Para o desenvolvimento das ações clínicas e educativas, utilizam-se tanto a estrutura da Unidade de Saúde do Bairro Ariri, composta por dois equipamentos odontológicos, macas e autoclave, como os espaços sociais que envolvam a comunidade em seu cotidiano (por exemplo, creches, asilos, escolas, praças, associações comunitárias e culturais). Também servem de apoio as Unidades Básicas de Saúde nas comunidades da Vila de Santa Maria e da Vila de Maruja, bem como os espaços escolares.

\section{ATIVIDADES}

Realiza-se um levantamento epidemiológico em que são investigados o índice CPOD, necessidade de tratamento e uso e necessidade de prótese, de acordo com a recomendação da Organização Mundial de Saúde [I7].

As pessoas também são convidadas a responder um questionário para caracterização socioeconômica e sobre conhecimentos, hábitos e condutas relacionados à saúde bucal, sendo posteriormente encaminhadas, segundo suas necessidades, para atividades de educação em saúde bucal e de escovação supervisionada, tratamento preventivo, adequação de meio bucal, tratamento periodontal, cirurgia oral menor ou encaminhamento para tratamento nas UBS do município.

Os atendimentos odontológicos promovidos pela equipe do Projeto Cananéia são pactuados com a Secretaria Municipal de Saúde.

Outras atividades, como análise dos teores de flúor na água de abastecimento público da comunidade, também podem ser incorporadas em função de interesses comuns.

\section{ASPECTOS ÉTICOS}

Previamente ao início das ações, a comunidade é informada e esclarecida quanto aos objetivos do Projeto Cananéia e seus membros são convidados a participar após a assinatura do termo de consentimento livre e esclarecido [2].

Os dados coletados durante a permanência da equipe são registrados em diário de campo e em fichas de levantamento. 


\section{RESULTADOS ESPERADOS}

Ao possibilitar que os estudantes vivenciem a saúde em seu sentido ampliado e percebam a determinação social do processo saúde-doença, cria-se uma oportunidade transdisciplinar de apreensão do conhecimento.

As Diretrizes Curriculares Nacionais para os cursos de Odontologia [3] apontam para a necessidade de mudanças importantes nos projetos pedagógicos, nos ambientes de prática e nas relações com os serviços de saúde e com as comunidades, assim como enfatizam a necessidade de formar um profissional apto a atender as demandas da população brasileira e em consonância com o Sistema de Saúde vigente [Io,7].

Participar de projetos de extensão desde o início da faculdade aproxima o aluno de graduação da realidade sobre a condição de saúde da população e sensibiliza-o sobre a importância das práticas em programas de prevenção e promoção de saúde, fornecendo subsídios para uma melhor formação acadêmica [I3].

É importante, entretanto, que essa participação não se restrinja a projetos voluntários de extensão, mas que seja fomentada ao longo de sua formação acadêmica. Para tanto, as instituições de ensino superior de todo o país, responsáveis por essa formação, deverão ter papel pró-ativo e serem os sujeitos desta ação.

Teixeira e Paim [I6] já percebiam a necessidade da reformulação do perfil dos profissionais, dos processos pedagógicos e também da política educacional em saúde. O processo de mudança foi recentemente estimulado pela implantação, por parte do Ministério da Saúde, em parceria com o Ministério da Educação, do Programa de Reorientação da Formação dos Profissionais de Saúde - Pró-Saúde [4].

As instituições inseridas nesse processo anteveem uma escola integrada ao serviço público de saúde, que responda às necessidades concretas da população brasileira na formação de recursos humanos, na produção de conhecimentos e na prestação de serviços, para o fortalecimento do Sistema Único de Saúde [II].

A discussão sobre a questão da saúde bucal como um problema coletivo de responsabilidade social passa pela necessidade de relacioná-la com o processo de participação da sociedade civil organizada nas decisões políticas e sociais, na formação de políticas de saúde bucal, na alocação e distribuição de recursos e nas decisões dos conselhos de saúde.

Para que haja o desenvolvimento de estratégias adequadas às realidades vivenciadas, é fundamental compreender a importância de priorizar a construção de ações coletivas junto à comunidade, na perspectiva da educação popular em saúde, em detrimento das ações unicamente assistencialistas.

Atuar nas comunidades estimula a pensar o cuidado em saúde focado na saúde da comunidade e não apenas nos escolares, identificando os segmentos populacionais que apresentam maiores riscos a causas específicas de adoecer, priorizando a atuação em regiões com desequilíbrio entre a demanda e a oferta de serviços de assistência odontológica e buscando soluções para melhorar a qualidade de vida da população.

\section{AGRADECIMENTOS}

Agradecemos a toda a equipe técnica que torna possível a concretização e o aperfeiçoamento do Projeto Cananéia: Maristela Vilas Boas Fratucci, Carlos Botazzo, Camila de Barros Gallo, Rebeca Silva de Barros, Rodrigo Elias de Oliveira, Carolina Mott de Arruda Fabrício, Nicole Erbert Ferrari.

\section{REFERÊNCIAS BIBLIOGRÁFICAS}

[I] BERBEL, N. A. N. A problematização e a aprendizagem baseada em problemas: diferentes termos ou diferentes caminhos? Interface Comunicação, Saúde, Educação, v. 2, n. 2, p. I39-I54, 1998.

[2] BRASIL. Ministério da Saúde. Conselho Nacional de Saúde. Comissão Nacional de Ética em Pesquisa. Normas para pesquisas envolvendo seres humanos (Resolução no I96/96 e outras). 2 ed. Brasília: Ministério da Saúde, 2003.

[3] BRASIL. Ministério da Educação. Conselho Nacional de Educação. Câmara de Educação Superior. Resolução CNE-CES n. 3, de I9 de fevereiro de 2002. Institui as Diretrizes Curriculares Nacionais dos Cursos de Farmácia e Odontologia. Diário Oficial da União. Brasília/DF, Seção I, p. IO, 4 de março de 2002.

[4] BRASIL. Portaria Interministerial n. 2.IoI, de 3 de novembro de 2005. Institui o Programa Nacional de Reorientação da Formação Profissional em Saúde Pró-Saúde - para os Cursos de graduação em Medicina, Enfermagem e Odontologia. Diário Oficial 
da União. Brasília/DF, n. 2I2, Seção I, p. III, 4 de novembro de 2005 .

[5] GNES. Estabelecimento de Saúde do Município: CANANEIA. Disponível em: <http://cnes.datasus. gov.br/Lista_Es_Municipio.asp?VEstado=35\&VCod Municipio $=350990 \&$ NomeEstado $=\mathrm{SAO} \% 2 \mathrm{OPAU}-$ LO>. Acesso em: 28 jun. 2007.

[6] DATASUS. Indicadores do Pacto de Atenção Básica 2006. Disponível em: <http://w3.datasus.gov.br/ datasus/datasus.php?area=359AiB 374 GiDoEoF35 9Gi8HoIIJdiL2IMoN\&VInclude=../site/infsaude. php\&VObj=http://tabnet.datasus.gov.br/cgi/siab/ pacto2006/pacmap.htm>. Acesso em: 20 jun. 2007.

[7] DIAS, M. G; PRESTA, A. A.; GAVA-SIMIONI, L.; SOUZA, P. B.; SALIBA, N. A. Currículo inovador em Odontologia: considerações a respeito. Saúde em Debate, v. 29, n. 69, p. 72-77, 2005.

[8] FEUERWERKER, L.; MARSIGLIA, R. Estratégias para mudanças na formação de RHs com base nas experiências IDA/UNI. Divulgação em Saúde para Debate, n. I2, p. 24-28, I996.

[9] INSTITUTO SOGIOAMBIENTAL. Vale do Ribeira: patrimônio da humanidade. Disponível em: <http://www.socioambiental.org/inst/camp/Ribeira/ vale>. Acesso em: 28 jun. 2007.

[io] MORITA, M. G.; KRIGER, L.; CARVALHO, A. C. P.; HADDAD, A. E. Implantação das diretrizes curriculares nacionais em Odontologia. Maringá: Dental Press/Abeno/Opas/MS, 2007.

[iI] MORITA, M. G.; KRIGER, I.; GASPARETTO, A.; TANAKA, E. E.; HIGASI, M. S.; MESAS, A. E.; IWAKURA, M. L. I.; ALVANHAM, D. Projeto Prósaúde odontologia: relato das atividades iniciais em universidades do estado do Paraná. Esp. Saúde, v. 8, n. 2, p. 53-57, 2007.

[12] PEIC. Ilha do Cardoso - Paraíso da vida marinha no Atlântico Sul. Disponível em: <http://www.cananet. com.br/peic/>. Acesso em: 28 jun. 2007.

[i3] PIZATTO, E.; GARBIN, G. A. S.; GARBIN, A. J. I.; SALIBA, N. A. O papel do professor no ensino odontológico. Saúde em Debate, v. 28, n. 66, p. 52-57, 2004.

[I4] SÃO PAULO. Universidade de São Paulo. Resolução n. 4940 , de 26 de junho de 2002. Baixa o Regimento de Cultura e Extensão Universitária da Universidade de São Paulo, definindo e regulamentando as atividades de cultura e extensão universitária. Diário Oficial do Estado. São Paulo, 3 de julho de 2002.

[I5] SEADE. Perfil municipal. Disponível em: <http:// www.seade.gov.br/produtos/perfil/perfil.php>.
Acesso em: 28 jun. 2007.

[i6] TEIXEIRA, C. F.; PAIM, J. S. Políticas de formação de recursos humanos em saúde: Conjuntura atual e perspectivas. Divulgação em Saúde para Debate, n. I2, p. I9-23, I996.

[i7] WORLD HEALTH ORGANIZATION. Oral health surveys: basic methods. 4 ed. Geneva: WHO, I997. 


\section{ANEXO}

\section{TABELA 1}

Distribuição dos moradores, por bairros, segundo grupos etários

Cananéia - 2007

\begin{tabular}{|c|c|c|c|c|}
\hline GRUPOS ETÁRIOS & ARIRI & $\begin{array}{l}\text { ILHA DO } \\
\text { CARDOSO }\end{array}$ & SANTA MARIA & TOTAL \\
\hline Menores de I ano & IO & 7 & o & I7 \\
\hline I a 4 anos & I7 & I5 & 7 & 39 \\
\hline 5 a 6 anos & 20 & I2 & $\mathrm{I} 2$ & 44 \\
\hline 7 a 9 anos & 3I & 20 & 27 & 78 \\
\hline IO a I4 anos & 60 & 34 & 38 & 132 \\
\hline I5 a I9 anos & 53 & 25 & 29 & 107 \\
\hline 20 a 39 anos & I32 & I06 & 76 & $3 \mathrm{I} 4$ \\
\hline 40 a 49 anos & 35 & 48 & I 8 & IOI \\
\hline 50 a 59 anos & 38 & 30 & 24 & 92 \\
\hline 60 anos e mais & 57 & 38 & 26 & I $2 \mathrm{I}$ \\
\hline Total & 453 & 335 & 257 & I.045 \\
\hline
\end{tabular}

